\title{
La ville palimpseste de Louis Sébastien Mercier. Spectralité, histoire et imaginaire temporel
}

\author{
Geneviève Boucher \\ Université d'Ottawa
}

Dans les interstices du discours rationaliste des Lumières se développe, dans le dernier tiers du XVIIIe siècle, un imaginaire de la revenance qui s'accompagne d'une forme d'esthétisation de la mort. Au cœur de cette sensibilité qui relève à la fois de l'esthétique gothique ${ }^{1}$, de la poétique des ruines et du goût pour

\footnotetext{
${ }^{1}$ The Castle of Otranto, a Gothic Story d'Horace Walpole, considéré comme le premier roman gothique, paraît en Angleterre en 1764 et connaît un succès fulgurant.
} 
un sublime du terrible ${ }^{2}$ se côtoient des représentations funèbres et des préfigurations apocalyptiques complaisamment inquiétées. Au-delà du renouveau esthétique, les figures fantastiques qui peuplent les œuvres se chargent d'une fonction heuristique et permettent de repenser la manière dont l'homme se rapporte au monde qui l'entoure : comment il le comprend, comment il le rêve, comment il le construit. Si les spectres occupent dans cet imaginaire une fonction particulière, c'est notamment parce qu'ils servent à penser le temps et à représenter les relations complexes que le présent entretient avec son passé. "Ghosts, in a sense, are history", écrit David Ratmoko (2006, p. 1) : ces voyageurs de l'au-delà condensent les tensions temporelles ambiantes et mettent en lumière les aspects les plus problématiques du rapport à l'histoire. Personnages transitoires, les fantômes qui hantent les textes sont des figures privilégiées pour effectuer le passage entre deux modes d'appréhension du temps : c'est pourquoi ils jouent un rôle aussi important à la fin du XVIIIe siècle, alors que l'on passe, selon des rythmes divers, de l'ancien régime d'historicité au régime moderne (Hartog, 2003).

L'œuvre panoramique de Louis Sébastien Mercier (17401814) offre un bon échantillon de ces phénomènes de revenance textuelle et des questions relatives à l'imaginaire historique qui y sont liées. Dans le Tableau de Paris, publié entre 1781 et 1788, puis dans Le Nouveau Paris, publié en 1798, l'auteur poursuit une double finalité : saisir le présent sur le vif pour en proposer une nouvelle lecture et faire l'histoire de ce même présent afin de comprendre comment il se rapporte ou

2 Pour Edmund Burke, «la terreur est [...] le principe qui gouverne le sublime» (p. 122). 
s'affranchit du passé. En interrogeant la fonction des revenants, "réels » et métaphoriques, qui peuplent les deux œuvres, on tentera de déterminer ce qui se joue dans leur présence textuelle, c'est-à-dire quelles tensions philosophiques, politiques ou idéologiques se donnent à lire dans les représentations de la revenance. Comme les deux œuvres sont séparées par l'abîme que creuse 1789 , on cherchera également à saisir les inflexions de ces représentations autour de cette coupure historique qui transforme l'imaginaire temporel en même temps que le système politique.

\section{L'épaisseur temporelle du panorama urbain}

De par son ancrage empirique, l'œuvre panoramique de Mercier semble exclure la fantasmagorie spectrale qu'on trouve dans d'autres œuvres du polygraphe comme les Songes et visions philosophiques (1788) ou Mon Bonnet de nuit (1784), toutes deux contemporaines du Tableau de Paris. De surcroît, l'imaginaire temporel qui se déploie dans le Tableau le rend, en théorie, incompatible avec tout phénomène de revenance. Le temps du Tableau n'est pas celui de la nostalgie, mais bien celui de la course contre la montre : animé par l'ambition de peindre Paris sous son jour le plus neuf, Mercier se condamne lui-même à une incessante réactualisation. Ce qui donne son impulsion au Tableau de Paris est sa fuite perpétuelle vers l'avant pour rattraper un présent qui se dérobe à l'écriture. Dans cet univers qui carbure à la contemporanéité, il n'y a apparemment pas de place pour les morts, et encore moins pour les revenants.

Or, si Mercier, au fil de ses promenades, cherche à saisir sur le vif le présent de la capitale, il lui est impossible d'ignorer 
ce qui compose ce présent. Dans une démarche que l'on pourrait qualifier d'archéologique ou de géologique, il déterre, sous la surface actuelle, les différentes strates temporelles qui y sont enfouies. Par exemple, il se montre fasciné par le mélange, dans le sol parisien, de matières humaines appartenant à de multiples couches temporelles. À plusieurs reprises dans le Tableau, il s'amuse à calculer le nombre de morts inhumés dans le sol de la capitale :

Dans l'espace de cent années, il faut que deux millions cinq cent mille individus déposent leurs ossements et leurs chairs alcalisées sur un point de six mille toises de circonférence; et dans cet espace, trente cimetières suffisent pour recevoir ce grand nombre de cadavres.

Nous avons dit que l'on déposait dans le cimetière des Innocents, situé dans le quartier le plus habité, près de trois mille cadavres par année. On y enterrait des morts depuis Philippe le Bel. Dix millions de cadavres au moins se sont dissous dans un étroit espace. (Mercier, 1994a [1781-1788], t. I, p. 645 et t. II, p. 732)

Le passé, dans sa matérialité la plus brute, se prolonge dans le présent, phénomène amplifié par le contraste entre le nombre considérable de cadavres et l'exiguïté de l'espace. Ailleurs, Mercier fait l'inventaire du bétail consommé annuellement par les Parisiens et dont les carcasses doivent être ensevelies : à ce nombre impressionnant (plus de six cent mille, toutes espèces confondues), s'ajoutent les vingt-deux mille dépouilles humaines qui peuplent annuellement le sol de la capitale :

Quatre-vingt-douze mille bœufs, vingt-quatre mille vaches, cinq cent mille moutons, voilà la consommation annuelle de la capitale. Calculez le nombre que cela fait au bout de cent ans. Joignez-y vingt-deux mille dépouilles mortelles pour les cimetières, et voyez si cette terre est engraissée, et comme elle doit abonder un jour en terre calcaire, produit égal, hélas ! des 
ossements humains et des ossements d'animaux. (Mercier, 1994a [1781-1788], t. II, p. 720)

Ces ossements, en se décomposant, « engraissent » le sol de la capitale, de telle sorte que les restes du passé, loin d'être de pures abstractions, forment le sous-sol de la capitale. L'histoire de la ville, l'histoire des générations qui y ont successivement vécu, est pensée à travers une métaphore géologique : elle est d'abord et avant tout une histoire du sol parisien.

En plus d'être historiques, les enjeux de ce mélange organique sont cosmologiques. Mercier s'était déjà montré fasciné, dans L'An 2440, par l'amalgame des chairs en décomposition: l'un des sages du XXVe siècle considérait les tombeaux « comme autant de creusets brûlants où la matière se fond et se dissout, où l'or s'épure et se sépare à jamais du vil métal» (Mercier, 1999a [1771], p. 159). L'imaginaire de l'alchimie se mêle ici à celui de la métempsycose pour sublimer la mort et la décomposition. La société de L'An 2440, qui croit en la réincarnation, est littéralement peuplée de l'esprit des morts : son évolution dépend de la transmission intergénérationnelle et, par conséquent, de l'absence de coupure radicale entre les vivants et les trépassés (Hamel, 2005). Loin d'être inquiétante, cette confusion de débris humains s'inscrit dans une cosmologie selon laquelle la matière organique circule dans l'univers, se régénère et se purifie. Comme l'a montré Paola Vecchi (1989), Mercier croit qu'une quantité égale de vie circule en permanence, prenant successivement différentes formes. La mort n'exclut pas l'être du cycle de la vie : elle est au contraire une étape essentielle par laquelle le vivant se régénère. C'est donc tout naturellement que, sous la plume de Mercier, les disparus s'intègrent au tableau de cette vaste capitale dont ils forment en quelque sorte le magma. 
On trouvait déjà chez Lavater, dont les travaux ont grandement inspiré Mercier, l'idée que le caractère d'une personne peut être déchiffré à travers sa physionomie, ses rides, ou, plus largement, à travers les différentes marques du passé qui s'impriment sur son visage et qui rendent ce passé à la fois visible et lisible. Selon une logique semblable, chez Mercier, l'identité des lieux parisiens, leur existence présente, est inséparable de leur histoire, de telle sorte que bien voir la ville, c'est aussi savoir lire les « rides » du Paris actuel, c'est voir le passé se profiler sous le présent, c'est faire émerger à la surface les couches temporelles enfouies et les peupler de fantômes. Ils sont nombreux, ces fantômes, dans le Tableau de Paris: Charlemagne, Philippe Auguste, Charles IX, Henri IV, Louis XIV, quelques reines, des ministres, des académiciens, des régicides - Ravaillac, Damiens - , puis une série de spectres anonymes - des "ombres jésuistiques", des prisonniers de Vincennes ou de la Bastille ou encore des squelettes sans nom exhumés du cimetière des Innocents. Ces spectres apparaissent au détour d'une place, à la vue d'une statue ou d'un monument, ils envahissent momentanément l'espace de la ville et font revivre leur époque dans le décor défiguré du Paris actuel.

Ils sont également porteurs d'un secret, d'une vérité longtemps enfouie dans les limbes de l'histoire officielle. Mercier s'exclame au sujet des morts exhumés du cimetière des Innocents : "Quelle histoire sortirait de cette enceinte, si les morts pouvaient parler! Que dit la nôtre en comparaison de tous ces faits oubliés et de ces divers caractères effacés dans la nuit des ténèbres? Nous ne savons rien sur nos ancêtres. » (Mercier, 1994a [1781-1788], t. II, p. 732) Les « faits oubliés » ensevelis dans la « nuit des ténèbres » se présentent comme les «caractères effacés » d'un livre, désormais illisible, dont les 
mots auraient été estompés. Tel un palimpseste, la ville est un manuscrit à demi effacé sur lequel des signes nouveaux sont apposés en permanence. Le présent s'écrit sur une surface qui n'est jamais vierge et, même si les signes du passé ont partiellement disparu, leur présence spectrale ne se laisse pas oublier, du moins, pas pour celui qui sait voir. Or, c'est précisément la tâche de l'écrivain-promeneur, cet expert du regard, que de voir, au-delà des couches superficielles, l'immense part de caché qui gît au cœur de ce monde que tous, à tort, croient connaître; c'est à lui qu'il revient de faire parler cette matière mi-morte mi-vive qui, autrement, serait restée muette. À la fatalité de l'histoire trouée, irrémédiablement perdue, s'oppose ainsi le désir de ressaisir le monde dans sa totalité en débusquant les fossiles du temps. Tout se passe comme si, pour redonner vie aux «caractères effacés » et réparer les erreurs de l'histoire officielle, il fallait, précisément, faire parler les morts. Dans les Songes et visions philosophiques, le cadre fantastique permet à l'auteur de faire revenir toute une série de morts célèbres qui, par leur discours, viennent rétablir la vérité. Parmi ces voyageurs de l'au-delà se trouve notamment Sémiramis, reine fondatrice de Babylone, à laquelle le narrateur fait subir un interrogatoire :

- Avez-vous en effet mis à mort votre époux Ninus ? - Non. L'histoire le dit. — L'histoire ment. — Mais M. de Voltaire a fait une tragédie là-dessus, et vous donne des remords. - Les tragédies sont des romans. - Et la voix de l'univers qui vous accuse? - L'univers sera désabusé. - Et quand-? - Quand le jour nécessaire pour la vérité sera venu.

À ces mots, la tête devint plus pesante; elle s'échappa de mes mains, et retomba dans son coffre. (Mercier, 2005 [1788], p. 98)

Comme les morts du cimetière des Innocents que Mercier déterre dans le Tableau de Paris, le fantôme de la reine 
babylonienne connaît des vérités cachées qui risquent de démentir l'histoire officielle, une histoire qui se voit reléguée au rang de construction culturelle en étant associée à la fiction littéraire par l'évocation ironique de la tragédie de Voltaire.

Si la spectralité implique la cohabitation des temporalités, on peut se demander comment ce feuilletage temporel, qui repose sur la rétention d'un certain passé, se conjugue au temps linéaire du progrès qui domine l'imaginaire des Lumières. Pour Jean-François Hamel, l'on aurait tort d'interpréter ce recours aux spectres comme un désir univoque de retour dans le passé, car la mise en scène de revenants sert surtout au présent à se représenter comme tel et peut même être considérée comme une condition paradoxale de l'idéologie du progrès :

La poétique du progrès entérine [...] la rupture de la tradition qui sépare les morts des vivants - le présent se détache du passé pour se projeter vers l'avenir - alors même qu'elle a pour condition de possibilité la transmission de blocs de passé d'une génération à l'autre - le présent se ressaisit du passé pour profiter des progrès antérieurs. (Hamel, 2006, p. 48)

Aucun progrès n'étant envisageable en dehors de cette chaîne générationnelle, c'est précisément parce que le dépassement est inséparable de l'accumulation que le modèle progressiste implique une forme d'épaisseur temporelle. La frontière entre les vivants et les morts doit donc se garder d'être trop étanche.

\section{Spectres et gestion du passé dans l'imaginaire révolutionnaire}

Cette intégration du passé au présent devient toutefois problématique après la Révolution, alors que l'idéologie de la table rase invite à prendre ses distances avec le modèle linéaire 
et, plus largement, avec toute forme de survie du passé dans le présent. Mercier ne renonce pas pour autant à peupler le présent des spectres du passé, mais ce passé n'est plus le même : il s'est considérablement rétréci. Alors qu'il couvrait, dans le Tableau, l'ensemble de ce qu'on appellera bientôt l'Ancien Régime (voire davantage, plongeant ses racines dans le passé lutécien et médiéval de la ville), il ne correspond plus, dans Le Nouveau Paris, qu'à la décennie précédant la Révolution, soit précisément la période couverte par le Tableau. Tout se passe comme si la seconde œuvre s'écrivait sur les caractères effacés de la première. Mercier l'annonce, dès l'ouverture :

Je ne marche plus dans Paris que sur ce qui me rappelle ce qui n'est plus. Bien m'a pris de faire mon tableau en douze volumes. Car s'il n'était pas fait, le modèle est tellement effacé qu'il ressemble au portrait décoloré d'un aïeul mort à l'hôpital et relégué dans un galetas. (Mercier, 1994b [1798], p. 31)

L'ensemble du Tableau est présenté comme une couche temporelle à jamais révolue, comme une histoire effacée sur laquelle marche le promeneur urbain. Une fois de plus, c'est la figure du palimpseste qui s'impose à travers les images du « modèle effacé » et du "portrait décoloré ». Dans Le Nouveau Paris, c'est le livre (le Tableau) qui sert à pallier l'oubli; c'est lui, avec ses douze volumes, qui permet de recomposer le passé, et non plus le «réel», qui a coupé tous les liens avec l'Ancien Régime. Là où, dans le Tableau, Mercier retrouvait dans la topographie urbaine un passé qui «était», il ne voit plus, dans Le Nouveau Paris, qu'un passé qui « n'est plus ».

Alors qu'à peine dix ans se sont écoulés depuis la cassure révolutionnaire, Mercier, de façon tout à fait étonnante, présente le Paris d'Ancien Régime comme une société en tous 
points coupée du présent, comme un monde radicalement étranger dont les rouages seraient incompréhensibles pour les contemporains. Cette altérité est amplifiée par la multiplication de notations explicatives visant à souligner la distance. Mercier écrit, par exemple :

La sœur du roi [...] n'avait d'autre crime que celui de sa naissance (pour parler le langage du temps).

Le Dauphin de France (car c'est le titre qui appartenait jadis à l'héritier présomptif de la couronne) [...]. (Mercier, 1994b [1798], p. 328 et 329)

L'on peut difficilement imaginer que les lecteurs de Mercier, en 1798, ne sachent pas ce qu'est un Dauphin de France. Mercier construit complaisamment la distance en feignant de s'adresser à une postérité pour qui les privilèges liés à la naissance ou les titres royaux seraient un jargon énigmatique. C'est pour cette postérité fictive qu'il exhume ce passé encore très récent. Mais en faisant cela, en recouvrant d'un voile d'étrangeté tout ce qui est antérieur à 1789, il amplifie la coupure et radicalise sa prise de distance avec ce passé monarchique que les révolutionnaires vouent à l'anathème. Paradoxalement, l'exhumation fantasmatique n'a pour but que d'accentuer l'inhumation qu'est en train d'accomplir, non sans peine, le présent, comme si l'historicisation anticipée devait parvenir à enterrer ce passé honni. Comme l'écrit Michel de Certeau, «le discours sur le passé a le statut d'être le discours du mort», ce qui fait de l'écriture historienne "l'équivalent des cimetières dans les villes; elle exorcise et avoue une présence de la mort au milieu des vivants » (1975, p. 73 et 122). Selon un double mouvement de présentification et d'exclusion, l'on ressuscite momentanément les morts parmi les vivants, mais l'on exorcise leur présence en les confinant à l'espace marginal du cimetière. S'il 
est ressuscité, le passé n'en est pas moins fortement altéré, car le propre de l'opération historiographique est justement de lui conférer un statut qui l'exclut de la mémoire vive du présent. L'écriture historique joue ainsi « le rôle d'un rite d'enterrement; elle exorcise la mort en l'introduisant dans le discours » (de Certeau, 1975, p. 139).

Même si Mercier affirme ne plus «marche[r] dans Paris que sur ce qui [lui] rappelle ce qui n'est plus », il ressuscite assez peu, dans Le Nouveau Paris, le passé monarchique, dont l'héritage est éminemment problématique. Lorsqu'il le fait, il veille à aménager des espaces marginaux qui empêchent les revenants d'envahir véritablement la cité. Dans un article du Journal de Paris d'octobre 1797, il rend compte de sa visite insolite au dépôt des Petits-Augustins, où sont entassés pêlemêle divers monuments ayant échappé au vandalisme révolutionnaire. Réunies par le hasard, les statues des personnages historiques s'animent d'une vie nouvelle sous l'effet de leur agencement improbable :

C'était véritablement là qu'il fallait lire les dialogues des morts de Fénelon et de Fontenelle, ou plutôt en composer de nouveaux. [...]

Un instant même je crus entendre la trompette du jugement dernier, et j'eus une vision d'Ézéchiel, il me semble que toutes ces statues confusément éparses et renversées allaient se relever et prendre une attitude vivante, mais dans cette égalité que conserveront tous les hommes devant le Juge éternel. (Mercier, 1994b [1798], p. 946-947)

Par leur mélange insolite, les personnages reviennent parmi les vivants pour être jugés - la double référence au jugement dernier est loin d'être anecdotique - et, par une injonction de l'Éternel, sont condamnés à l'égalité : descendus des piédestaux 
qui attestaient de leur supériorité, ils se voient assimilés au commun des mortels :

Tous les rangs, tous les costumes, toutes les couronnes étaient sous mes pieds; j'épargnais le visage et le sein des reines; les plus fameux personnages descendus de leur piédestal étaient redevenus à mon niveau; je pouvais toucher leur front, leur bouche, parler à l'oreille de Richelieu, et interroger Malebranche. (p. 946)

Là où la classification est absente, il ne règne aucune hiérarchie : les rois et les autres personnages importants sont désormais accessibles à quiconque souhaite les « interroger » et ils se distinguent à peine du promeneur qui les scrute. Mieux : l'ordre s'est renversé, car les «rangs», «costumes» et « couronnes » se trouvent «sous les pieds» du promeneur, qui est libre de les piétiner négligemment. Mercier utilise le même argumentaire pour commenter le vandalisme des tombes royales de Saint-Denis :

Tous ces rois qui semblaient devoir vaincre, au fond de leurs cercueils, la durée des siècles, vainement protégés par les simulacres des anges et des saints, et par les prodiges du ciseau, sentirent, après tant d'années de repos, les coups de cette incroyable révolution. (p. 944-945)

Nul ne peut échapper à la loi universelle du temps, qui rassemble l'humanité dans un destin commun, sans distinction de rang ni d'état. C'est là le nouvel ordre de la Révolution, qui présente comme égaux l'ensemble des citoyens et qui punit l'orgueil de ceux qui ont cru pouvoir s'élever au-dessus de cette égalité fondamentale.

Le «désordre sublime» (p. 948) qui fascine Mercier au dépôt des Petits-Augustins ne dure hélas que quelques semaines. Les objets du patrimoine national sont rapidement 
placés en ordre chronologique, rangés, classifiés pour former le Musée des monuments français, dirigé par Alexandre Lenoir et ouvert au public à l'automne 1795. Dans un article publié en juin 1798, Mercier déplore cette froide mise en ordre qui a fait rentrer les morts dans leur tombe. Il a alors une idée originale pour ranimer le tout :

Apportez des flambeaux; que tout ceci s'anime, que le cimetière disparaisse, avec l'entourage de ces chapelles mortuaires; que ce musée soit fermé le jour et qu'il ne soit permis d'y entrer que la nuit; apportez des flambeaux. Que ce peuple de statues symétriquement rangées devienne mouvant et colossal; que je parcoure à la lueur des torches cette ville de morts, ces mausolées, les tombeaux de tous ces personnages dont la vue me redit l'histoire; que les ombres de Charlemagne et de Louis IX montent jusqu'aux voûtes, animent tous les autres monuments, et que ces grandes ombres soient encore escortées par celles de Moïse, d'Isaïe et des autres prophètes, dont le sublime extasie ma mémoire. (Mercier, 1994b [1798], p. 951-952)

En imaginant cette scène baroque, où la poétique du mélange côtoie l'esthétique sépulcrale et en reprenant une série de topoi $\mathrm{du}$ roman gothique, Mercier traite les monuments du patrimoine national comme autant de fantômes, ce qui est d'autant plus significatif que l'une des préoccupations du moment est précisément de définir comment la société révolutionnaire peut se rapporter à ce passé problématique qu'elle a détruit, mais qu'elle tient néanmoins à conserver.

Il peut sembler contradictoire que Mercier tienne à ce point à faire revivre les grandes figures de l'Ancien Régime, lui qui, par ailleurs, se réjouit de ce qu'on ait détruit les tombes royales de Saint-Denis et que l'on ait placé les restes des rois dans une fosse commune. Mais justement, le musée présente cet avantage qu'il permet de conserver les vestiges du passé sans les intégrer à 
l'espace de la vie sociale. À partir du moment où un objet entre au musée, il perd sa valeur de monument, il se détache du monde pour exister dans cet espace marginal voué à la mémoire (Poulot, 1993). Pierre Nora, dans l'introduction des Lieux de mémoire, insiste sur la distinction fondamentale entre mémoire et histoire : il y a histoire dès lors qu'il n'y a plus de mémoire vivante, c'est-àdire dès lors que le présent se détache du passé comme le maillon d'une chaîne brisée. Une société s'invente des lieux de mémoire lorsqu'elle souhaite réactiver un passé révolu; elle crée ainsi les archives qui lui permettent de reprendre possession de ce passé pour expliquer l'époque présente :

Les lieux de mémoire naissent et vivent du sentiment qu'il n'y a pas de mémoire spontanée, qu'il faut créer des archives, qu'il faut maintenir des anniversaires, organiser des célébrations, prononcer des éloges funèbres, notarier des actes, parce que ces opérations ne sont pas naturelles. (Nora, 1984, p. XXIV)

Tout comme l'historicisation, la création d'un patrimoine national, sanctionnée par l'institution du musée, isole les différents objets du monde ambiant. Le geste muséal a ainsi la fonction paradoxale d'effacer ce que, précisément, il donne à voir : il retire du monde les objets patrimoniaux pour les immobiliser dans un statut de passé qui leur impose une forme de mort. En inventant le patrimoine national, les révolutionnaires ne font donc pas que se doter d'une mémoire collective : ils officialisent leur rupture avec l'Ancien Régime en mettant ce passé à distance.

\section{Les fantômes de la Terreur : de l'historiographie à la justice suprême}

Entre 1796 et 1798, au moment où Mercier écrit Le Nouveau Paris, un autre passé, celui de la Terreur, hante les 
contemporains, qui s'efforcent de l'historiciser pour mieux s'en détacher. Le souvenir traumatique de cette période sanglante - Mercier, emprisonné pour avoir soutenu les députés girondins, a bien failli y laisser sa tête - et le désir de régler ses comptes avec la politique montagnarde motivent pour une large part la rédaction du Nouveau Paris, dont l'une des visées est d'esquisser une histoire de la Révolution au sein de laquelle la Terreur ne serait qu'un dérapage funeste ${ }^{3}$. Après Thermidor, il semble impératif de purger la Révolution de son association infamante à la violence terroriste. On est obsédé par l'idée d'un possible retour des robespierristes et, plutôt que de chasser le spectre de la Terreur en l'excluant des représentations, on se met à ressusciter Robespierre pour lui faire un procès éternel. Mercier évoque le foisonnement, dans les mois suivant Thermidor, de pièces mettant en scène les événements les plus traumatiques de l'épisode révolutionnaire, comme pour exorciser la violence de la Terreur :

Ces revenants, ces spectres, qu'on évoque sur les théâtres, et qu'on se plaît à contempler, sont le reflet des journées révolutionnaires : le peuple se plaît, dans la fantasmagorie, à voir l'ombre de Robespierre; elle s'avance; un cri d'horreur s'élève, tout à coup sa tête est détachée de son corps, un terrible coup de tonnerre écrase le monstre; et des acclamations de joie accompagnent la détonante fulmination. (Mercier, 1994b [1798], p. 657)

L'«ombre de Robespierre" ne revient pas pour hanter le peuple, mais bien pour être jugée. D'ailleurs, la lame de la

\footnotetext{
${ }^{3}$ À ce discours consistant à faire de la Terreur une parenthèse dans le cours de la Révolution s'en oppose un autre, tenu notamment par les contrerévolutionnaires, selon lequel la Terreur serait indissociable des principes révolutionnaires mêmes qui, portés à leur paroxysme, ne pourraient que dégénérer dans la violence et la tyrannie. (voir Backzo, 1989, Gueniffey, 2000, et Mayer, 2000)
} 
guillotine qui tombe sur le tyran prend la forme d'un ordre tombé du ciel et son impact se dédouble en un « terrible coup de tonnerre », signe d'une justice divine cautionnant celle des hommes. En rejouant ainsi, sur le mode tragique, le supplice du tyran, on procède à une forme d'exorcisme collectif qui consiste à faire revivre temporairement l'esprit maléfique pour mieux jouir de son anéantissement définitif. C'est un peu le même phénomène qui se produit dans L'An 2440 où, par injonction de l'Éternel, le spectre de Louis XIV est condamné à errer sur les ruines de Versailles. Lorsque le narrateur, déambulant sur les vestiges du château, fait la rencontre de l'auguste fantôme, ce dernier lui confie, en pleurs :

Ah! malheureux! sachez que je suis ce Louis XIV qui a bâti ce triste palais. La justice divine a rallumé le flambeau de mes jours pour me faire contempler de plus près mon déplorable ouvrage... Que les monuments de l'orgueil sont fragiles... Je pleure, et je pleurerai toujours... Ah ! que n'ai-je su... (Mercier, 1999 [1771], p. 294)

Le spectre, qui appartient à la catégorie des «fantômes piaculaires » (Sangsue, 2011, p.108), a une fonction de réparation: il revient sur terre sur l'injonction d'un principe moral supérieur à l'histoire des hommes afin d'expier ses fautes et de subir la justice à laquelle il a échappé de son vivant. Comme dans le cas de Robespierre, la mise en scène du châtiment a l'effet d'un exorcisme : elle rétablit l'ordre dans la cité en purgeant le présent d'un épisode honteux de son histoire.

Mercier est loin d'être le seul, dans le contexte thermidorien, à ranimer le tyran pour rejouer son procès. Après la mort de Robespierre, plutôt que d'enfouir son souvenir, on se met à le ressusciter et à le faire parler dans différents textes - 
un testament imaginaire au ton fortement ironique ou, encore, une série de lettres d'outre-tombe dans lesquelles l'Incorruptible gère sa postérité 4 . Il est significatif que, plutôt que de dénoncer simplement la politique montagnarde, ces textes s'ingénient à faire parler Robespierre à la première personne, comme si l'enjeu était bien de le faire revenir, de le rendre à nouveau présent dans l'espace de la cité... pour mieux l'en chasser. Ces textes d'aspect testamentaire posent de surcroît le problème de l'héritage, qui est à la base de tout phénomène de revenance. La crainte de voir resurgir la violence de la Terreur se cristallise dans l'image de Robespierre en monstre dont la tête aurait été coupée, mais dont la queue subsisterait à travers ses héritiers. Ces représentations tirent leur origine d'une rumeur voulant que Robespierre ait dit, avant de mourir, que l'on pouvait bien lui couper la tête, mais qu'il laissait sa queue (Baczko, 1989, p. 79). Une série de pamphlets $^{5}$ se mettent alors à circuler pour dénoncer la « queue » de Robespierre, c'est-à-dire ses anciens complices qui continuent à siéger à la Convention (Billaud-Varenne, Collot d'Herbois, Barère, etc.). Derrière ce débat, qui s'alimente d'une imagerie particulièrement éloquente, se trouve l'idée que Robespierre ne sera mort que lorsque tous ses continuateurs

\footnotetext{
${ }^{4}$ Testament de I.M. Robespierre, trouvé à la maison commune, 1794; La tête à la queue, ou Première lettre de Robespierre à ses continuateurs, 1794 et Rendez-moi ma queue, ou Lettre de Robespierre à la Convention nationale, 1794.

${ }^{5}$ Jean-Claude Méhée, La Queue de Robespierre, Paris, 1794 et Défends ta queue, Paris, 1794 (signés Felhémési); Couturier, La Queue de Robespierre écorchée, s.d.; La Pierre de touche jettée [sic.] sur les conspirateurs, ou Suite à La queue de Robespierre, 1794 [signé Raliobonsleujei]; Marie-Joseph Chénier, Ode à la calomnie, en réponse à La queue de Robespierre, 1794; Marie et Prévost, Réponse à la queue de Robespierre par un franc républicain, 1794; Saintomer, Jugement du peuple souverain, qui condamne à mort la Queue infernale de Robespierre, 1794.
} 
auront été exclus du gouvernement, comme si, pour tourner la page de la Terreur, il fallait d'abord enterrer tous ses morts.

Cet exorcisme, qui consiste à faire revivre temporairement le spectre du tyran, est également symptomatique d'une peur, d'une méfiance à l'égard de morts qui auraient été mal enterrés et qui, du coup, menaceraient de revenir. Cette crainte de la hantise trouve un écho dans un projet de loi sur les sépultures, auquel Mercier réagit fortement. Le polygraphe transcrit, dans Le Nouveau Paris, le discours qu'il a présenté au Conseil des Cinq-Cents en décembre 1796 pour s'opposer à la proposition de laisser aux proches des défunts la liberté de disposer à leur guise de leur dépouille. Pour Mercier, ce serait ouvrir la porte à de dangereux abus : il craint que les morts soient enterrés n'importe où, ou encore, que leurs cendres, conservées à la surface, ne finissent par envahir l'espace social. L'argumentation de Mercier se fonde sur deux idées présentées comme interdépendantes : 1 . l'incinération est contre nature, car elle empêche le cadavre de "retourner à la terre ", c'est-à-dire de se décomposer dans la Terre-mère dont il est issu; 2. les rites funéraires et la gestion des sépultures doivent être contrôlés par une instance centralisée, car la mort est un phénomène qui rattache l'humain au social autant qu'au divin. Privatiser les usages entourant la mort reviendrait donc à isoler l'individu des entités supérieures dont il relève (la société, la Nature) et auxquelles il est redevable. La vision matérielle et géologique impliquée par la cohabitation temporelle se prolonge dans une forme d'intemporalité divine : les cadavres « appartiennent indistinctement à la terre, qui leur a prêté ses éléments, et n'appartiennent qu'à elle » (Mercier, 1994b [1798], p. 809-810). Une fois privé de son âme, le corps ne demanderait qu'à rentrer dans la terre 
parce qu'il est fait pour s'y décomposer lentement et successivement, et par des lois physiques reconnues. C'est là qu'il accomplit la dette qu'il a contractée en naissant, et il n'est honorablement et utilement que là.

Vouloir brûler ce corps, comme le demande le rapporteur, est une erreur grossière, si ce n'est pas au fond un attentat physique, un sacrilège envers la nature; car c'est empêcher le reversement des matières composantes qui forment la nourriture, la richesse et la parure du globe. (p. 811)

Élément destructeur, le feu priverait la terre de «ce qu'elle a droit d'attendre pour la reproduction des végétaux et pour la formation des terres calcaires » (p. 811) et ferait de la mort un anéantissement plutôt qu'une régénération. Pour Mercier, l'immortalité de l'âme est inséparable d'une certaine immortalité du corps, laquelle exclut toute destruction définitive de la matière organique. L'on retrouve une fois de plus l'imaginaire cosmogonique selon lequel la matière circule à l'intérieur du Vivant, se recyclant périodiquement en prenant une forme tantôt minérale, tantôt végétale, tantôt animale ou humaine. Pour Mercier, bouleverser l'ordre de la nature en autorisant l'incinération et en dérégularisant les rites funèbres briserait non seulement l'harmonie cosmique, mais ferait courir le risque de sombrer dans l'anarchie d'un monde où les morts, mal enterrés, reviendraient hanter les vivants :

Les inhumations et sépultures tiennent tout à la fois à des rapports religieux, civils et politiques; et ces rapports sont si délicats, qu'il faut user de la plus grande sagesse pour les concilier. Prenons garde que les morts ne troublent le repos des vivants; cela s'est vu dans bien des pays; j'en atteste l'histoire, celle surtout des vampires; il y a dans le projet de quoi la renouveler; et les sépultures privées que l'on vous propose, je le crains avec quelque fondement, pourraient nous conduire à des troubles tout aussi imprévus. (p. 809) 
Le fait que Mercier fasse allusion aux vampires dans un discours présenté devant l'Assemblée législative est moins étonnant qu'il n'y paraît. En plus de hanter l'imaginaire social, les débats entourant l'existence des vampires animent la communauté scientifique européenne dans la première moitié $\mathrm{du}$ XVIIIe siècle. Les Dissertations sur les apparitions des anges, des démons et des esprits et sur les revenans et vampires et Hongrie, de Bohême, de Moravie et de Silesie du bénédictin Dom Augustin Calmet, exégète biblique reconnu, sont rééditées quatre fois entre 1746 et 1759 et traduites, dès leur première publication, en italien et en allemand, ce qui témoigne de l'intérêt que portent à ce problème les autorités savantes, médicales et politiques les plus respectées ${ }^{6}$ (Köpeczi, 1980, et Venturino, 2008). Mais au-delà de la question des vampires, ce que révèle le texte de Mercier est l'idée que les morts doivent être confinés à un espace marginal, celui du cimetière, lequel est régi par des lois civiles. Les enjeux sont les mêmes que ceux qui se sont déjà posés au sujet du musée: il est nécessaire d'assigner aux morts et aux représentants du passé une place qui leur est propre. L'imagination peut bien s'amuser à les ressusciter, mais cela ne peut se faire que dans un espace clos, en marge de la société.

Les spectres, chez Mercier, se rapportent à un ordre supérieur: ils sont les agents ou les victimes d'une justice divine qui condamne le vice, récompense la vertu et rétablit

\footnotetext{
${ }^{6}$ Si des savants comme Dom Calmet s'intéressent au phénomène dans une perspective scientifique, ils n'en concluent pas pour autant à l'existence des vampires. D'ailleurs, l'intérêt pour ces revenants buveurs de sang finit par s'essouffler à partir des années 1760. L'article «Vampire» de l'Encyclopédie range explicitement ces croyances dans la catégorie des superstitions, tout comme le fait Voltaire, en 1772, dans la neuvième partie des Questions sur l'Encyclopédie. À ce titre, la croyance de Mercier semble tout à fait hétérodoxe.
} 
l'équilibre social en rendant tous les hommes égaux dans la mort. Les revenants n'incarnent donc pas le chaos ni la disjointure d'un présent qui n'arriverait pas à coïncider avec lui-même: ils ont une présence rassurante en ce qu'ils sont porteurs d'un sens de l'histoire. La spectralité débouche sur un nouvel ordre du monde selon lequel l'histoire est placée sous la tutelle bienveillante d'une justice éternelle. Les spectres deviennent ainsi des médiateurs précieux qui permettent d'articuler le passé au présent en jouant à la fois sur la distance et sur l'intégration et qui, ultimement, incitent à poser sur l'histoire humaine le regard surplombant d'une instance supérieure.

\section{Bibliographie}

BACZKo, Bronislaw. (1989), Comment sortir de la Terreur. Thermidor et la Révolution, Paris, Gallimard.

BonNET, Jean-Claude (dir.). (1995), Louis Sébastien Mercier (17401814). Un hérétique en littérature, Paris, Mercure de France.

BURKE, Edmund. (2009 [1757]), Recherche philosophique sur l'origine de nos idées du sublime et du beau, Paris, Vrin, coll. "Bibliothèque des textes philosophiques", présentation, traduction et notes par Baldine Saint Girons. 
Certeau, Michel de. (1975), L'Écriture de l'histoire, Paris, Gallimard, coll. « Folio histoire ».

Gueniffey, Patrice. (2000), La Politique de la Terreur. Essai sur la violence révolutionnaire 1789-1794, Paris, Gallimard, coll. « Tel ».

HAMEL, Jean-François (2006), Revenances de l'histoire. Répétition, narrativité, modernité, Paris, Minuit, coll. «Paradoxe».

- . (2005), «Les uchronies fantômes. Poétique de l'histoire et mélancolie du progrès chez Louis Sébastien Mercier et Victor Hugo », Poétique, no 144, p. 429-441.

Hartog, François. (2003), Régimes d'historicité. Présentisme et expériences du temps, Paris, Seuil, coll. «La librairie du XXIe siècle ».

KöPECZI, Béla. (1980), «Un scandale des Lumières: les vampires », dans Raymond Trousson (dir.), Thèmes et figures du Siècle des Lumières. Mélanges offerts à Roland Mortier, Genève, Droz, p. 123-135.

KoSELLECK, Reinhart. (1990), Le Futur passé. Contribution à la sémantique des temps historiques, traduit de l'allemand par Jochen Hoock et Marie-Claire Hoock, Paris, Éditions de l'École des hautes études en sciences sociales.

MAYER, Arno J. (2000), The Furies: Violence and Terror in the French and Russian Revolutions, Princeton, Princeton University Press.

MALL, Laurence. (2011), « L'Avoir-été et le n'être-plus : le travail de la mémoire révolutionnaire dans Le Nouveau Paris (1798) de L. S. Mercier », $M L N$, vol. 126, no 4, p. 889-904. 
MÉCHOUlan, Éric. (2004), Pour une histoire esthétique de la littérature, Paris, Presses universitaires de France, coll. « L'interrogation philosophique».

MERCIER, Louis Sébastien. (2005 [1788]), Songes et visions philosophiques, Paris, Éditions Manucius, coll. "Littéra», texte établi, annoté et présenté par Jean-Claude Bonnet.

-. (1999a [1771]), L'An 2440, rêve s'il en fut jamais, Paris, La Découverte.

- (1999b [1784]), Mon bonnet de nuit, Paris, Mercure de France, édition établie sous la direction de Jean-Claude Bonnet.

- (1994a [1781-1788], Tableau de Paris, Paris, Mercure de France, coll. "Librairie du bicentenaire de la Révolution française », édition établie sous la direction de Jean-Claude Bonnet, 2 tomes.

-. (1994b [1798]), Le Nouveau Paris, Paris, Mercure de France, coll. "Librairie du bicentenaire de la Révolution française», édition établie sous la direction de Jean-Claude Bonnet.

NoRA, Pierre. (1984), «Entre mémoire et histoire: la problématique des lieux», dans Les Lieux de mémoire. I. La République, Paris, Gallimard, coll. « Bibliothèque illustrée des histoires », p. XV-XLII.

Poulot, Dominique. (1993), «Le patrimoine des musées : pour l'histoire d'une rhétorique révolutionnaire », Genèses, no 11, p. 25-49.

RATMOKO, David. (2006), On Spectrality. Fantasies of Redemption in the Western Canon, New York, Peter Lang. 
SANGSUE, Daniel. (2011), Fantômes, esprits et autres mortsvivants. Essai de pneumatologie littéraire, Paris, José Corti.

STALNAKER, Joanna. (2006), « The New Paris in Guise of the Old: Louis Sébastien Mercier from Old Regime to Revolution", Studies in Eighteenth-Century Culture, no 35, p. 223-242.

Stierle, Karlheinz. (2001), La Capitale des signes. Paris et son discours, traduit de l'allemand par Marianne Rocher-Jaquin, Paris, Éditions de la Maison des sciences de l'homme.

Turcot, Laurent. (2007), Le Promeneur à Paris au XVIIIe siècle, Paris, Gallimard, coll. « Le promeneur ».

VECCHI, Paola. (1989), «La balance et la mort: progrès et compensation chez Louis-Sébastien Mercier», Studies on Voltaire and the Eighteenth Century, 264, p. 905-908.

Venturino, Diego. (2008), « Dom Calmet, "historiographe des vampires"? », dans Philippe Martin et Fabienne Henryot (dir.), Dom Augustin Calmet. Un itinéraire intellectuel, Paris, éditions Riveneuve, p. 233-246.

\section{Résumé}

Si Louis Sébastien Mercier cherche, dans le Tableau de Paris (1781-1788), à saisir sur le vif la physionomie de la capitale, il est aussi appelé à déterrer le passé qui, à l'instar d'un palimpseste, demeure partiellement visible sous la surface actuelle. Une série de spectres, représentants de ce passé révolu 
mais réanimé, envahissent la ville et apportent sur leur époque un témoignage précieux. Une telle intégration du passé dans le présent devient toutefois problématique après la Révolution. Dans Le Nouveau Paris (1798), le passé monarchique n'est ressuscité que dans des espaces marginaux qui soulignent son altérité; quant au passé terroriste, il est exorcisé par la mise en scène des spectres de ses représentants qui reviennent parmi les vivants pour être soumis au jugement suprême de l'Histoire.

\begin{abstract}
If Louis Sébastien Mercier, in his Tableau de Paris (1781-1788), tries to take hold of the capital's physiognomy, the nature of his project bring him to exhume the city's past which, like a palimpsest, is partially visible under the present. A number of ghosts embody this ambiguous past: they haunt the city and carry around a secret that could contradict the official version of History. Such a coexistence of past and present is however highly problematic in the post-revolutionary context. In $L e$ Nouveau Paris (1798), the monarchic past is only resuscitated in marginal spaces that underline its otherness; as for the terrorist past, it is exorcised by bringing the Montagnard leaders back to life in order to submit them to the supreme judgement of History.
\end{abstract}

